\title{
ESPÉCIES DE MOSCAS-DAS-FRUTAS (DIPTERA: TEPHRITIDAE) ASSOCIADAS À CULTURA DA GOIABEIRA (PSIDIUM GUAJAVA LINNAEUS) EM FORTALEZA, CEARÁ
}

\section{A.P. Moura ${ }^{1} \&$ D.C.M. Moura ${ }^{2}$}

IUniversidadeFederal deLavras, Departamento deEntomologia, CP 3037, CEP 37200-000, Lavras, MG, Brasil. E-mail: alexandrepm@yahoo.com

RESUMO

O presente trabal ho foi real izado com o objetivo de se conhecer as espécies de moscas-dasfrutas (Diptera:Tephritidae) associadasàculturadagoiabeira (Psidium guajava Linnaeus) (Myrtaceae) em Fortaleza, Ceará, bem como sua flutuação populacional e análise quantitativa de sua população. As coletas desses tefritídeos foram realizadas, semanalmente, em um pomar de goiabeira localizado no campus do Pici, na Universidade Federal do Ceará, no período de novembro de 1999 a outubro de 2000. Utilizaram-se frascos caça-moscas tipo M cPhail contendo cerca de $50 \mathrm{~mL}$ desolução atrativa (glucosedemilho), na concentração de $100 \%$ para a captura dos insetos adultos. Para se determinar a associação entre a planta hospedeira e as espécies de tefritídeos deocorrência na área foram real izadas col etas sistemáticas defrutos duranteo mesmo período. Coletaram-se frutos maduros diretamente das árvores, bem como frutos recém-caídos. As espécies de moscas-das-frutas registradas para a cultura da goiabeira em Fortaleza foram A nastrepha sororcula Zucchi, A nastrephazenildae Zucchi eC eratitiscapitata (Wiedemann), estaúltima ocorrendo em maior número e durante praticamente todo o período de condução das coletas.

PALAVRAS-CHAVE: A nastrepha, Ceratitis, biodiversidade, Myrtaceae, ocorrência.

\section{ABSTRACT}

SPECIES OF FRUIT-FLIES (DIPTERA: TEPHRITIDAE) ASSOCIATED TO GUAVA CROP (PSIDIU M GU AJA VA LINNAEUS) IN FORTALEZA, CEARÁ, BRAZIL. Thegoal of this study was to learn about the species of fruit-flies (Diptera: Tephritidae) associated to guava crop (Psidium guajava Linnaeus) (Myrtaceae) in Fortal eza, Ceará, including population dynamicsand quantitative analysis of thepopulation. Collections of theseTephritidaewereaccomplished, weekly, in guava orchard located at the UniversidadeFederal do Ceará, from N ovember of 1999 to October of 2000. McPhail traps containing $50 \mathrm{~mL}$ of attractivesolution (corn glucose) at $100 \%$ wereused to capture theinsects. To determinetheassociation between the host plant and the species of Tephritidaein thearea, systemiccollections of fruitsweremadeduringthesameperiod. Ripefruitswerecollected directly from theplants as well as somenewly-fallen fruits. A nastrephasororcula Zucchi,A nastrepha zenildae Zucchi and Ceratitis capitata (Wiedemann) werethefruit-fly speciesassociated toguavacrop in Fortaleza. The fruit-flyC . capitata wasthemostabundantspecies, occurring practically during the whole period of this study.

KEY WORDS: A nastrepha, Ceratitis, biodiversity, Myrtaceae, occurrence.

\section{INTRODUÇÃO}

O Brasil é o terceiro maior produtor de frutas frescasdomundo, produzindo cercade 35 milhões de toneladas anual mente e gerando cerca de 4 milhões deempregos diretos, sendo queo $\mathrm{N}$ ordestebrasileiro destaca-secomo um dos maiores produtorese exportadores de frutas do país. Em relação à cultura da goiabeira( Psidium guajava Linnaeus), oBrasil produziu no ano de 2002 cerca de 389 mil toneladas, das quais a região Nordeste respondeu por aproximadamente 40\% dessetotal (A GRIANUAL, 2005).

A cultura da goiabeira experimentou um incre mento significativo na área plantada, passando de aproximadamente 14 mil hectares no ano de 2000 para cerca de 18 mil hectares plantados em 2002, enquanto quea produção, duranteo mesmo período, cresceu cerca de $65 \%$ (AgrianuAL, 2005). As exportações dessafruta, porém, não acompanharamomesmo ritmo decrescimento, sendo que uma das principais

2Universidade Federal de Lavras, Departamento de Engenharia A grícola, Lavras, MG, Brasil. 
causas para essa situação éa presença depragas nas áreas cultivadas, entre el as as moscas-das-frutas.

As moscas-das-frutas são consideradas importantes pragas da fruticultura mundial, causando perdas significativas à produção e limitando o livre trânsito defrutas devido às restrições impostas pelos países importadores (MALAVASI, 2000). No Brasil, as espéciesdemoscas-das-frutas deimportânciaeconômica pertencem a 4 gêneros: A nastrepha, Bactrocera, Ceratitis eR hagol etis (Diptera: Tephritidae). Noentanto, do ponto devista agrícola, apenasCer atitiscapitata (Wiedemann) e 7 das 94 espécies de A nastrepha que ocorrem no Brasil são as moscas-das-frutas economicamente importantes no país (ZuccHI, 2000a).

A pesar dograndeconhecimento acercadas diversas espécies de tefritídeos que causam prejuízos à fruticulturabrasileira, poucoaindasesabesobresuas plantashospedeiras. Trabal hosrealizadospor ZucCHI (2000b) evidenciaram que para $56 \%$ das espécies de A nastrepha assi naladas no Brasil, não há registros da relação com plantas hospedeiras, visto queamaioria dos levantamentos érealizada com armadilhas contendo atrativos alimentares, o que impossibilita associá-las com segurança aos seus hospedeiros.

Assimsendo, o presentetrabalho tevepor objetivo conhecer as espécies de moscas-das-frutas (Diptera: Tephritidae) associadas à cultura da goiabeira em Fortaleza, Ceará, bemcomosuaflutuaçãopopulacional e análise quantitativa de sua população, tanto por meio de coletas utilizando-se de armadil has contendo atrativo alimentar, quanto por meio de coleta e incubação de frutos infestados.

\section{MATERIAL E MÉTODOS}

$\mathrm{O}$ trabal ho foi realizado em área contíguaà $\mathrm{H}$ orta Didática e de Pesquisa da Universidade Federal do Ceará (UFC), localizada no campus do Pici, em Fortaleza, Ceará. Para a coleta de insetos adultos foram instaladas quatro armadilhas tipo McPhail (SALLES, 1995) em um pomar de goiabeiras (cultivadas em espaçamento $6 \mathrm{~m} \times 6 \mathrm{~m}$ ) deaproximadamente 5 anos deidade, distribuídasuniformementeemumaáreade cerca de um hectare. As armadilhas foram distribuídas sobre as plantas a uma altura de aproximadamente $160 \mathrm{~cm}$ do solo esempreao lado norteda copa, demodo a protegêlas da incidência deraios solares. Utilizou-se como atrativo al imentar a glucose de miIho a $100 \%$.

Semanalmente, no período de $1^{\circ}$ de novembro de 1999a 27 de outubro de 2000, realizaram-seas coletas das moscas capturadas nas armadilhas, ocasião em queasolução atrativafoi renovadadecadaumadelas, perfazendo um total de 52 avaliações realizadas no campo. Frutos maduros foram coletados ao acaso em
6 plantas escol hidas al eatoriamente, bem como frutos recém-caidossob suas copas, conformesua disponibilidade no momento das avaliações semanais.

OsinsetossemanalmentecapturadosnasarmadiIhas foram acondicionados em frascos de vidro (etiquetados) providos de tampa, de aproximadamente $8 \mathrm{~cm}$ de altura $\times 3 \mathrm{~cm}$ de diâmetro, contendo álcool a $70 \%$ de modo a conservá-los até o momento de sua identificação específica. Em seguida, foram transportadosatéo Laboratório deEntomologiaAgrícola da UFC, também localizado no campus do Pici.

Os frutos col etados foram transportados para o mesmo laboratório, ondeforam contados, pesadose, posteriormente, colocadosemplacas dePetri de $20 \mathrm{~cm}$ de diâmetro x $2,5 \mathrm{~cm}$ de profundidade contendo vermiculita. Foram, então, mantidosatemperaturade aproximadamente $29 \pm 30 \mathrm{C}$, umidaderelativade $75 \pm$ $10 \%$ efotofase de $12 \mathrm{~h}$, por um período de 14 dias, de modo a permitir a saída das larvas e conseqüente pupação na vermiculita. Decorrido esse período os frutosforamremovidoseas placas dePetri colocadas em gaiolas confeccionadas em acrílico $(40 \mathrm{~cm}$ de altura $\times 30 \mathrm{~cm}$ de largura $\times 30 \mathrm{~cm}$ de comprimento) contendo orifícios decerca de $15 \mathrm{~cm}$ de diâmetro nas lateraisenaporta. Essesorifíciosforamfechadoscom tecido tipo filó, de modo a facilitar a aeração no interior das gaiolas, onde as placas permaneceram por 7 dias, paraquepudesseocorrer a emergência de adultos demoscas-das-frutas. A pósa emergência, os adultos foram coletados, mortos, contados e acondicionadosemfrascos devidroetiquetados, demaneira semel hanteà descritaanteriormente, atéadatadesua identificação específica.

A identificação dos tefritídeos foi realizada por especialista, com auxílio de chaves analíticas para identificação demoscas-das-frutas, ao nível deespé cie, no Laboratório deEntomologiaA grícola da UFC. Depois deidentificados, os insetos capturados tanto por meio dearmadilhas, como os emergidos defrutos incubados foram contados em relação ao número de insetos de cada espécie encontrada, sendo os dados obtidos utilizados para a confecção dos gráficos da flutuação populacional dessas espécies durantetodo o período de execução do estudo.

De posse dos dados numéricos das moscas coletadas, consideraram-se as variações populacionais das espécies associadas à cultura da goiabeira, levando-se em conta sua densidade populacional. Vincularam-se, ainda, os dados obtidosduranteapesquisaadadosmeteorológicosfornecidos pela Estação de Meteorologia do Centro de CiênciasA grárias daUFC ( $3^{\circ} 33^{\prime}$ Se38 $33^{\circ}$ W), distante cerca de $1,1 \mathrm{~km}$ do local de realização do estudo.

Realizou-se, também, a análise faunística das espécies de tefritídeos coletadas, baseando-se em índices sugeridos por Silveira Neto et al. (1976), KreBS 
(1978) e LUdWIG \& ReYNoLds (1988), avaliando-sefreqüência, constância, riqueza e dominância.

A freqüência $\left(p_{i}\right)$ diz respeitoàrazão entreonúmero deindivíduos deuma espécie $\left(n_{i}\right)$ e o número total de indivíduos da amostra $\left(N_{i}\right)$. No que se refere à constância (C) deumadada espécie, a mesmaédeterminada pela relação entre o número de amostras contendo essaespécie ( $p$ )eo númerototal deamostras tomadas $(\mathrm{N})$, sendoqueas espécies, quanto àconstância, podem ser classificadas em (1) espécies constantes (presentes em mais de $50 \%$ das amostras), (2) espécies acessórias (presentes em $25 \%$ a $50 \%$ das amostras) e (3) espécies acidentais (presentes em menos de $25 \%$ das amostras). A riqueza (S), por sua vez, correspondeao número total deespécies observadas na comunidade, enquanto quea dominância de uma espécie é determinada baseando-se em sua freqüência ena riqueza das espécies; uma espécie, portanto, é considerada dominante quando apresenta freqüência superior $\mathrm{a} 1 / \mathrm{S}$.

\section{RESULTADOSE DISCUSSÃO}

Verificou-sebaixariqueza deespécies demoscasdas-frutas (Diptera:Tephritidae) ocorrendo naculturada goiabeiranocampus doPici,emFortaleza, Ceará, durante o período de realização do presente estudo.


to por meio de armadilhas como pela incubação de frutos), sendo elas A nastrepha sororcula Zucchi, A nastrepha zenildae Zucchi e Ceratitis capitata (Wiedemann). Dentreessas espécies, A . sor orcula eA . zenildae são consideradas não-dominantes e aci dentais, enquanto $C$. capitata éconsiderada dominantee constante. Essaúltimaespécieapresentou freqüência de $96,75 \%$ (Tabela 1 ).

Os baixos índices de constância obtidos para A . sororcula $(13,46 \%)$ e A. zenildae $(7,69 \%)$ (Tabela 1 ) sugerem quea ocorrência dessas espécies em pomares de goiabeira na cidade de Fortaleza é considerada esporádica. Possivel mente, essefato ocorra em função da competição dessas espécies com a mosca-domediterrâneo, C . capitata.

Acredita-sequeos baixosvalores demoscascapturadasedediversidadeespecífica(riquezadeespécies) na área em estudo possam estar relacionados à eliminação de frutos de goiabeira caídos, em virtude dos tratosculturaisrealizadosnessaárea, fatoresessesque podem ter impedido o desenvolvimento eproliferação de uma maior população desses tefritídeos no local. URAмото (2002) sugerequearealização sistemáticade tratos culturais em áreas cultivadas com frutíferas, eliminandofrutoscaídosaosolo,implicanaerradicação de sítios de oviposição para as moscas-das-frutas, diminuindo assim sua população.

Baseando-se nos val ores de freqüência e constância deC . capitata observadosnopresenteestudo(Tabela 1), sugere-sequeestaespécie, apesar deexótica, encontra-sealtamente adaptada às condições climáticas da CidadedeFortaleza, oquedemonstraainda, seu maior sucesso competitivo em comparação às demaisespécies(A. sororcula eA .zenildae). Possivel mente, asmai ores freqüência e constância verificadas para $C$. capitata estejam relacionadas, também, ao fato dequefoi constatadaa infestação defrutos "devez" por essa espécie, o quenão foi observado para as demais.

Levantamentos realizados no ano de 1997 por meio de inspeções diretas, e por armadilhas d'água, Jackson eM cPhail, em 6 grandes áreas do interior do Estado do Ceará, também evidenciaram a ocorrência de A . sororcula e A . zenildae em cultura da goiabeira; entretanto, a mosca-do-mediterrâneo C . capitata apenas foi constatada atacando as culturas do cafeeiro e da tangerineira (SA LES \& GonçALVES, 2000).

No que diz respeito à flutuação populacional de $C$. capitata, verificou-sequeessaespéciedetefritídeoocorreu durantetodo o período decondução do presenteestudo, exceto no mês deagosto de2000(Fig. 1). A credita-seque a não ocorrência da mosca-do-mediterrâneo no mês de agostode2000tenhasidoinfluenciadapelabaixadisponibilidadedefrutos degoiabeira duranteo mesmo mês, quando foi coletado apenas 0,6 kg de frutos (Fig. 2), limitando, assim, a existência de sítios de oviposição.

Tabela 1. A náli sefaunística (riqueza, freqüência, dominância econstância) das espécies demoscas-das-frutas (Diptera: Tephritidae) col etadas em pomar de goiabeira no campus do Pici, Fortal eza, Ceará, de novembro de 1999a outubro de 2000.

\begin{tabular}{lcccc}
\hline Espécies(Riqueza) & $\mathrm{n}_{\mathrm{i}}$ & Freqüência(\%) & Dominância* & Constância(\%)** \\
\hline A nastrepha sororcula & 23 & 2,67 & $\mathrm{n}$ & $13,46 \mathrm{ac}$ \\
A nastrepha zenildae & 5 & 0,58 & $\mathrm{n}$ & $7,69 \mathrm{ac}$ \\
Ceratitis capitata & 833 & 96,75 & $\mathrm{~d}$ & $92,31 \mathrm{c}$ \\
\hline Total & 861 & - & - & - \\
\hline *d =dominante; $n$ =não-dominante. & & & \\
** $\mathrm{c}=$ espécieconstante; ac =espécieacidental. & & &
\end{tabular}




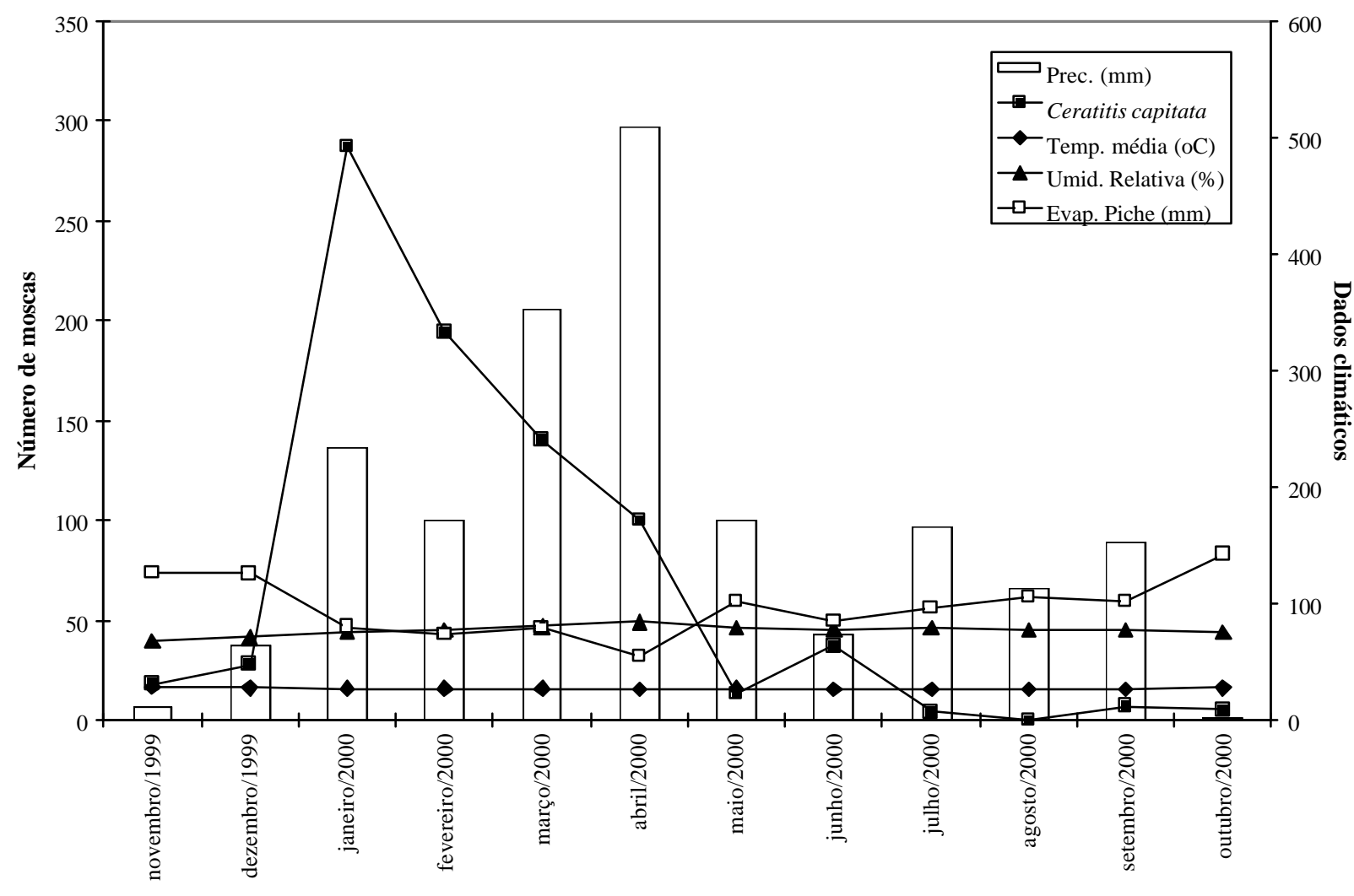

Meses

Fig. 1- Flutuação populacional deadul tos deC eratitiscapitata coletados em pomar degoiabei ra local izado nocampusdo Pici, em Fortal eza, Ceará, evariações dosfatores cl imáticos [precipitação total $(\mathrm{mm})$, temperatura média $\left({ }^{\circ} \mathrm{C}\right)$, umidade relativa (\%) eevaporação total em evaporímetro de Piche (mm)], de novembro de 1999 a outubro de 2000.

Os níveis populacionais dessa espécie de insetopraga mantiveram-se mais elevados durante o primeiro quadrimestre de 2000, sendo que seu pico populacional ocorreu no mês de janeiro, quando foram coletados cerca de $35 \%$ do total de moscas dessa espécie. Duranteos meses dejaneiro, fevereiro, março e abril de 2000, foram coletados 287, 194, 140 e 100 espécimes, respectivamente. A olongo desses 4 meses coletaram-se cerca de $86 \%$ de todos os indivíduos capturados durantetodo o período de real ização do presente trabal ho (Fig. 1).

A credita-se que a maior ocorrência de C . capitata durante o primeiro quadrimestre do ano 2000 , possa estar relacionada tanto à grande disponibilidade de frutos duranteesteperíodo (Fig. 2), quantoaosfatores climáticos, notadamenteà precipitação. Verificou-se, porém, que a medida que se registrou aumento gradativo na precipitação, observou-seredução tambémgradativanonúmerodemoscascoletadas(Fig. 1). Diante dos resultados obtidos pode-se sugerir quea ocorrência dessa espécie de moscas-das-frutas em Fortalezaencontra-se, ao menosem parte, relacionada à precipitação.
BATEMAN (1972) verificou que a abundância de moscas-das-frutas podeser influenciada por diversos fatores tanto bióticos quanto abióticos. Segundo A LujA (1994), estudosrealizadosem pomarescomerciais têm revelado que as flutuações populacionais de adultos de moscas-das-frutas estão diretamente relacionadas a dois complexos de variáveis: disponibilidade de plantas hospedeiras e condições climáticas.

Observou-se, também, que o número de moscasdas-frutas coletadas apenas por meio da incubação de frutos foi bastante elevado. Em geral, as maiores infestações desses tefritídeos em frutos de goiabeira ocorreram durante o primeiro quadrimestre de 2000 (Fig. 2), quando também ocorreu o pico populacional deC . capitata (Fig. 1). Paraessamesmaespécie, durante os meses de janeiro, fevereiro, março e abril do mesmo ano verificaram-se índices de infestação de 45,5; 87,1; 31,2 e 40,4 adultos/ kg, respectivamente. Para as espécies de A nastrepha coletadas durante o estudo, os índices de infestação foram de 4,3 e 0,3 adultos/ kg, para osmeses demarço emaio do ano de 2000, respectivamente (Fig. 2). 




Fig. 2 - Flutuação da coleta de frutos e índices de infestação (adultos/ kg) de moscas-das-frutas (Diptera: Tephriti dae) coletadas por mei o da incubação defrutos de goiabei ra em pomar local izado nocampus do Pici, em Fortaleza, Ceará, de novembro de 1999 a outubro de 2000.

Com base nos dados constantes na Figura 3, que ilustraaflutuação populacional demoscas-das-frutas do gêneroA nastrepha, verifica-sequeaocorrênciadesse gêneronaculturadagoiabeiraemFortal ezarestringiuse aos meses de março, abril e maio de 2000. Não foi registrada a ocorrência de um único indivíduo desse gênero infestando a cultura da goiabeira nos demais meses em que foram realizadas avaliações.

Assimcomosugerido paraC .capitata acredita-seque ascondiçõesdimáticas, principal mente, aprecipitação, tenham influenciado na ocorrência e flutuação populacional das espécies de A nastrepha detectadas na cultura da goiabeira em Fortaleza, Ceará. N o entanto, 0 fator maisimportantequepareceterinfluenciadoessas variáveis biológicas foi a competição interespecífica coma mosca-do-mediterrâneo, C . capitata.

Estudos desenvolvidos por A lujA \& al .(1996), porém, sugerem queessetipo decomportamento sejaconsideradonormal, vistoqueemboradiversasespédiesdetefritídeos estejam presentes em um pomar, apenas uma ou duas espécies representam mais de $90 \%$ de todas as moscas coletadas em armadilhas. A elevada dominância de apenasumaou duasespéciesdemoscas-das-frutastam- bémfoi verificadapor diversosoutrospesquisadores, em trabal hossimilaresreal izadosnoBrasil (KovALESKI, 1997; Veloso, 1997; Garcia \& Corseull, 1998; Uramoto \& al., 2003; Uramoto \& al ., 2004; Araújo et al ., 2005).

Duranteomês demarço de2000foramcapturados 20 espécimes deA . sororcula e 4 deA .zenildae. No mês de abril, apenasA .zenildaefoi registrada, com a ocorrência de um único indivíduo, enquanto que no mês demaio do mesmo ano, coletou-seapenasA .sororcula (3 indivíduos) (Tabela 2).

As duas espécies de moscas-das-frutas do gênero A nastrepha coletadas no presenteestudo são consideradas, segundo ZuCCHI (2000a), particularmente importantes do ponto de vista econômico para a fruticultura brasileira, sendo que A . zenildae, segundo CANAL et al. (1998)éconsideradaimportantepragaparaaculturada goiabeira também no nortedo estado de Minas Gerais.

Essas constatações vêm confirmar a importância quetais espécies deA nastrephatem paraafruticultura nordestina, principalmenteparaaculturadagoiabeira, visto que se trata de um hospedeiro preferencial paraestas ediversas outras espécies detefritídeos de ocorrência no Brasil. 


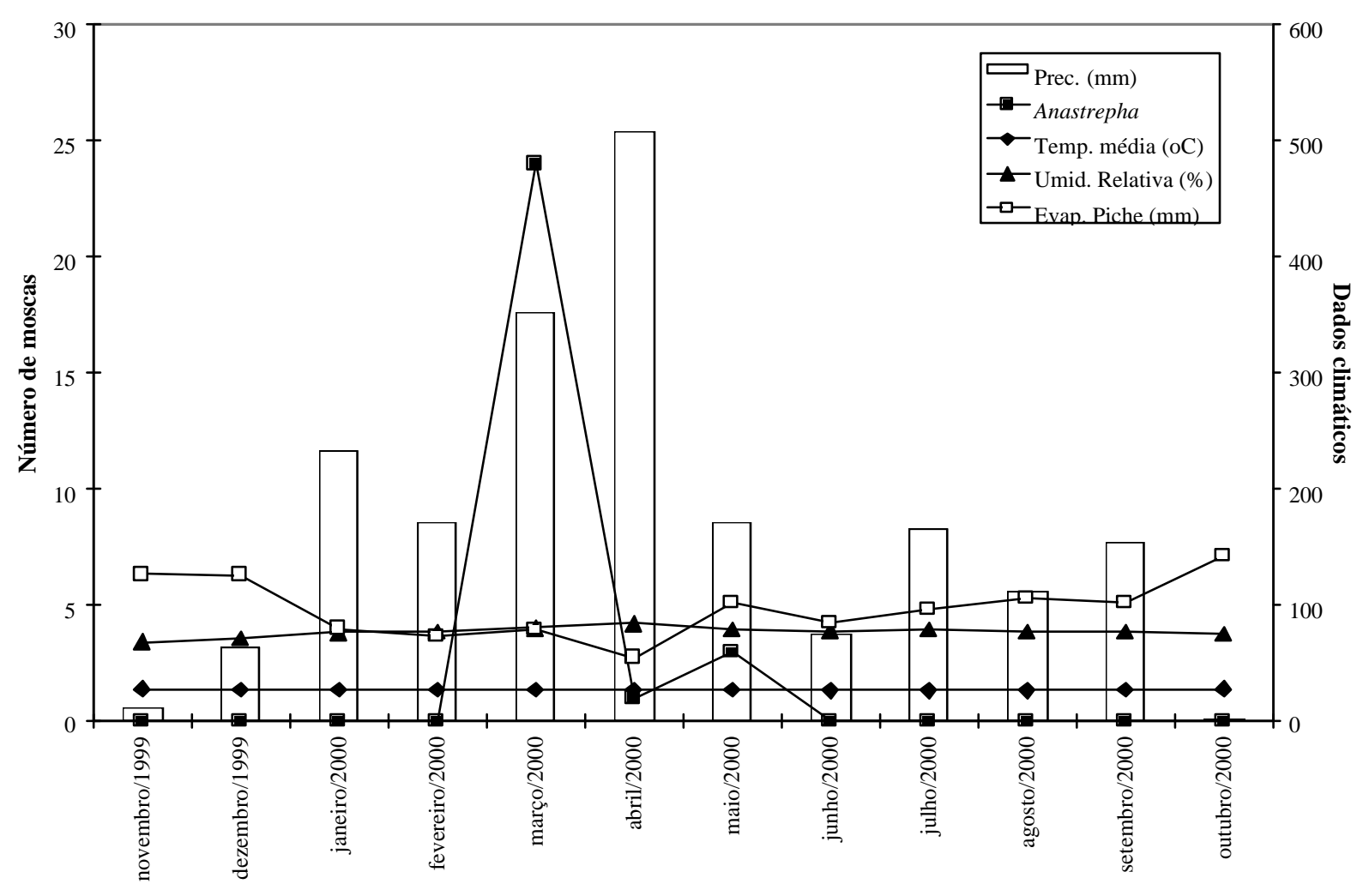

Meses

Fig. 3- Flutuação populacional deadul tos deA nastrepha spp. coletados em pomar degoiabeira local izado nocampusdo Pici, em Fortal eza, Ceará, evariações dosfatores cl imáticos [precipitação total ( $\mathrm{mm}$ ), temperatura média $\left({ }^{\circ} \mathrm{C}\right)$, umidade relativa (\%) eevaporação total em evaporímetro de Piche (mm)], de novembro de 1999 a outubro de 2000.

Tabela 2 - Espécies de A nastrepha (Diptera: Tephritidae) coletadas na cultura da goiabeira no campus do Pici, em Fortaleza, Ceará, denovembro de1999a outubro de2000.

\begin{tabular}{lccr}
\hline Mês/ ano & \multicolumn{2}{c}{ Espécies } & Total \\
\cline { 2 - 3 } & A. sororcula & A . zenildae & \\
\hline novembro/ 1999 & 0 & 0 & 0 \\
dezembro/ 1999 & 0 & 0 & 0 \\
janeiro/ 2000 & 0 & 0 & 0 \\
feverei ro/ 2000 & 0 & 0 & 0 \\
março/ 2000 & 20 & 4 & 24 \\
abril/ 2000 & 0 & 1 & 1 \\
maio/ 2000 & 3 & 0 & 3 \\
junho/ 2000 & 0 & 0 & 0 \\
julho/ 2000 & 0 & 0 & 0 \\
agosto/ 2000 & 0 & 0 & 0 \\
setembro/ 2000 & 0 & 0 & 0 \\
outubro/ 2000 & 0 & 0 & 0 \\
\hline Total & 23 & 5 & 28 \\
\hline
\end{tabular}

Todas as espécies de moscas-das-frutas capturadaspor meio dearmadilhasforam, também, coletadas por meio daincubação defrutos degoiabeira, assegurando, assim, que essas espécies ocorrem na Cidade de Fortaleza infestand a cultura da goiabeira.

Valesal ientar queé de suma importância a associação correta de uma espécie de moscas-das-frutas com seu fruto hospedeiro, o queapenas podeser feito deformasegura, por meio daincubação detaisfrutos. Segundo Zucchı (2000a), a associação equivocada de uma espécie demoscas-das-frutas com um fruto hospedeiro pode acarretar sérios problemas quarentenários.

\section{CONCLUSÕES}

Os resultados obtidos no presente estudo permitem concluir que:

1. A nastrepha sororcula, A nastrepha zenildae e Ceratitis capitata são as espécies de moscas-das-frutas que atacam a cultura da goiabeira em Fortaleza, Ceará; 2. Ceratitis capitata é considerada uma espécie dominante e constante, enquanto A nastrepha sororcula e A nastr epha zenil dae são não-dominantes eacidentais; 3. A mosca-do-mediterrâneo Ceratitis capitata é a espécie mais freqüenteem goiabei ras na Cidadede 
Fortaleza, representando $96,75 \%$ do total demoscas coletadas;

4. A espécie Ceratitis capitata ocorre durante praticamente todo o ano em goiabeiras na Cidade de Fortaleza;

5. O período de maior ocorrência de moscas-dasfrutas em goiabeiras na Cidade de Fortaleza é o primeiro quadrimestre do ano.

\section{AGRADECIMENTOS}

AoConselho Nacional deDesenvolvimentoCientífico eTecnológico - CN Pq, pela concessão de bolsa de estudos ao primeiro autor e à Dra. Niédja G. G. Gonçalves pela identificação das espécies de A nastr epha coletadas durantea realização do presente estudo.

REFERÊNCIAS

Agrianual: A nuário da agriculturabrasileira. São Paulo:FNP Consultoria \& Comércio, 2005. p.314-318.

A LujA, M. Bionomics and management of A nastrepha. A nnual Review of Entomology, v.39, p.155-178, 1994.

Aluja, M.; Celedonio-Hurtado, H.; Liedo, P.; Cabrera, M.; Castillo, F.; Guillén, J.; Rıos, E. Seasonal population fluctuations and ecological implications for management of A nastrepha fruit flies (Diptera: Tephritidae) in commercial orchards in Southern Mexico. Journal of E conomic Entomology, v.89, n.3, p.654667, 1996

Araújo, E.L.; Medeiros, M.K.M.; Silva, V.E.; Zucchi, R.A. Moscas-das-frutas(Diptera:Tephritidae) no semi-árido do Rio Grande do N orte: plantas hospedeiras e índices deinfestação. N eotropical Entomology, v.34,n.6, p.889-894, 2005.

Bateman, M.A. The ecology of fruit flies. A nnual Review of Entomology, v.17, p.493-581, 1972.

Canal, N.A.; Alvarenga, C.D.; Zucchi, R.A. Níveis de infestação de goiaba por A nastrepha zenildae Zucchi, 1979 (Dip., Tephritidae), em pomares comerciais do N ortedeM inas Gerais. A nais da SociedadeEntomológica do Brasil, v.27, n.4, p.657-661, 1998.

GARCIA, F.R.M. \& CoRSEUIL, E. Flutuação populacional de A nastrephafraterculus (Wiedemann) eC eratitiscapitata (Wiedemann) (Diptera, Tephritidae) em pomares de pessegueiro em Porto Alegre, Rio Grande do Sul. Revista Brasileira deZoologia, v.15, n.1, p.153-158, 1998.

Kovaleskı, A. Processos adaptativos na colonização da maçã (M alus domestica L.) por A nastrepha fraterculus (W ied.) (Diptera: Tephritidae) na região de Vacaria, RS. 1997. 122p. Tese (Doutorado) - Instituto de Biociências, UniversidadedeSão Paulo, São Paulo, 1997.
KREBS, C.J. Ecology: theexperimental analysis of distribution and abundance. New York: Harper \& Row, 1978. $678 p$.

LudWIG, J.A. \& ReYnolds, J.F. Statistical ecology: a primer on methods and computing. New York: John Wiley, 1988. 337p.

MalavasI, A. Áreas-livres ou de baixa prevalência. In: MALAVASI, A. \&ZucchI, R.A. (Eds.). M oscas-das-frutasde importância econômica no Brasil: conhecimento básico e aplicado. Ribeirão Preto: Hollos, 2000. p.175-181.

Sales, F.J.M. \& Gonçalves, N.G.G. Ceará. In:Malavasi ,A.\& Zucchı, R.A. (Eds.). M oscas-das-frutas de importância econômicanoBrasil: conhecimento básico eaplicado. Ribeirão Preto: Hollos, 2000. p.217-222.

SALLES, L.A.B. (Ed.). Bioecologia e controle da mosca-das-frutas Sul-americana. Pelotas:EMBRAPA/ CPACT, 1995. 58p.

Silveira Neto, S.; Nakano, O.; Barbin, D.; Villa Nova, N.A. $M$ anual de ecologia dos insetos. São Paulo: Agronômica Ceres, 1976. 419p.

Veloso, V.R.S. Dinâmica populacional de A nastrepha spp. e Ceratitis capitata (W ied., 1824) (D iptera: Tephritidae) nos cerrados de Goiás, Goiânia, G 0. 1997. 115p. Tese (Doutorado) - EscoladeA gronomia, UniversidadeFederal deGoiás, Goiânia, 1997.

URAMoto, K.; WaLDER, J.M.M.;ZucchI, R.A. Biodiversidade demoscas-das-frutas do gênero A nastrepha (Diptera, Tephritidae) no campus da ESALQ-USP, Piracicaba, São Paulo. Revista Brasileira de Entomologia, v.48, n.3, p.409-414, 2004.

Uramoto, K.; Walder, J.M.M.; Zucchi, R.A. Flutuação populacional de moscas-das-frutas do gênero A nastrepha Schiner, 1868 (Diptera, Tephritidae) no Campus "Luiz de Queiroz", Piracicaba, São Paulo. Arquivos do Instituto Biológico, São Paulo, v.70, n.4, p.459-465, 2003. Disponível em: łttp:/ / www. biologico.sp.gov.br/ arquivos/ v70 4/ uramoto.pdf>. Acesso em: 2 nov. 2005

URAmoto, K. Biodiversidade de moscas-das-frutas do gênero Anastrepha (D iptera: T ephritidae) no campus Luiz de Q ueiroz, Piracicaba, São Paulo. 2002. 85p. Dissertação (M estrado) - EscolaSuperior deAgricultura"Luizde Queiroz", Universidade de São Paulo, Piracicaba, 2002.

Zucchı, R.A. Taxonomia. In: Malavasi, A. \& Zucchi, R.A. (Eds.). M oscas-das-frutas de importância econômica no Brasil: conhecimento básico e aplicado. Ribeirão Preto: Hollos, 2000a. p.13-24.

Zucchı, R.A. Espécies de A nastrepha, sinonímias, plantas hospedeiras eparasitóides. In:MALAVASI, A.\&ZUCCHI, R.A. (Eds.). M oscas-das-frutas de importância econômica no Brasil : con hecimento básico eaplicado. Ribeirão Preto: Hollos, 2000b. p.41-48.

Recebido em: 2/ 12/ 05

A ceito em: 9/ 2/ 06 